\title{
Day-to-day variability in the occurrence characteristics of Sq focus during d-months and its association with diurnal changes in the Declination component
}

\author{
S. Alex and M. M. Jadhav \\ Indian Institute of Geomagnetism, New Panvel, Navi Mumbai 410 218, India \\ (Received June 22, 2005; Revised September 21, 2007; Accepted October 2, 2007; Online published November 30, 2007)
}

\begin{abstract}
Scrutiny of the geomagnetic observations of the Declination component ' $D$ ' from equatorial and low-latitude locations in the Indian longitude revealed anomalous variations during d-months. Examination of the Quiet day $(\mathrm{Ap} \leq 7)$ variations in the Declination component $(Y$ in $\mathrm{nT}=H \sin D)$ and Horizontal component $(H)$ during the d-months (November, December, January, February) of the low solar activity year 1977 revealed the salient features associated with the day-to-day changes observed in Sq current system over the latitude chain in the Indo-Russian longitude. Diurnal variations in the east-west component $Y$ and north-south component $H$ were used to depict the effect of changes in the Sq current pattern for a selected set of Quiet days, which are classified into normal and abnormal days based on the trend in the variation of the Declination component. Normal days designated in the study clearly showed the expected pattern of variation in $\Delta Y$ (eastward-directed field) in the morning hours and westward-directed field in the afternoon hours, and diurnal variation of $\Delta H$ simultaneously showed the presence of northern $\mathrm{Sq}$ focus within the low-latitude range. In contrast, the abnormal days projected by the variation in $\Delta Y$ and $\Delta H$ on certain days of d-months showed an almost complete absence of the focus formation within the expected latitude extent. Day-to-day variability characteristics of the Declination component discussed in the study are illustrated in relation to modifications in the expected Sq current system by the invasion of the prevalent southern hemisphere current whorl to the northern hemisphere during the winter months. Some of the anomalous changes observed in the day-to-day variation in the latitudinal pattern of $\Delta Y$ and $\Delta H$ components are also interpreted as suggesting an influence of high-latitude magnetospheric current systems on certain quiet days.
\end{abstract}

Key words: Declination component, eastward field, abnormal day, Sq current.

\section{Introduction}

The daily variation in the magnetic field at the Earth's surface during the geomagnetic quiet condition is known to be generated by solar heating and results in tidal winds in the E-region of the ionosphere, where the magnitude of conductivity is appreciable. The ionospheric current system is responsible for these variations on solar quiet $(\mathrm{Sq})$ days. Hasegawa (1960) pointed out that the day-to-day changes in the position of the focal latitude during quiet magnetic conditions would influence the pattern of the Sq current system. Schlapp (1968) studied the day-to-day variability of Sq based on the IGY data obtained from a global network. He suggested that the day-to-day variability of Sq is due to the variations in dynamo driving force rather than variations in conductivity. Noticeable changes in the seasonally varying characteristics of the Sq current system, the location of the focal latitude and the complexity associated with the absence of the Sq current vortex, especially during the d-months in the Indian longitude sector, have been studied by various researchers (Tarpley, 1973; Patil et al., 1983; Campbell and Matsushita, 1982; Rastogi, 1993; Campbell

Copyright (c) The Society of Geomagnetism and Earth, Planetary and Space Sciences (SGEPSS); The Seismological Society of Japan; The Volcanological Society of Japan; The Geodetic Society of Japan; The Japanese Society for Planetary Sciences; TERRAPUB et al., 1991; Mazaudier, 1993). Patil et al. (1983) noted some relevant features of the $\mathrm{Sq}$ current vortex at locations in the vicinity of focal latitude at Gulmarg. Remarkable changes in the summer-winter variation pattern in the eastward field were reported by Rastogi (1993) based on the magnetic field component data for the period 1975-1976. Campbell et al. (1993) studied the track of the Sq current focus on quiet days of 1976 and 1977 and reported cases in which the Sq vortex disappeared during the winter months of both years. However, Alex et al. (1992), in their study of the month-to-month variation characteristics of the Declination $(D)$ component for the d-months of 1986, 1987 and 1988, demonstrated complex day-to-day variation patterns in $D$ and the Horizontal component $(H)$. Their result is in contrast to those of Rastogi (1993) and Campbell et al. (1993) who discussed the absence of the focus of the Sq current system during d-months. Oyama et al. (2000) proposed the mechanism of anomalous electron temperature in local winter months as a factor contributing to the behaviour of the solsticial asymmetry of the Sq focus.

To date, most of the studies that have examined the variability features of the eastward component have been based on average seasonal variations. In the study reported here, a significantly varying magnitude and phase in the diurnal variation of Declination is used as a guiding factor in as- 
Table 1. Geographic and geomagnetic co-ordinates.

\begin{tabular}{|c|c|c|c|c|c|c|}
\hline \multirow[t]{2}{*}{ Stations } & \multirow[t]{2}{*}{ Code } & \multicolumn{2}{|c|}{ Geographic } & \multicolumn{2}{|c|}{ Geomagnetic } & \multirow[t]{2}{*}{ Dip $\left({ }^{\circ}\right.$ Lat. $)$} \\
\hline & & Latitude & Longitude & Latitude & Longitude & \\
\hline TRIVANDRUM & TRD & $8^{\circ} 29^{\prime} \mathrm{N}$ & $76^{\circ} 57^{\prime} \mathrm{E}$ & $0.88 \mathrm{~S}$ & $148^{\circ} .24$ & $0.2^{\circ} \mathrm{N}$ \\
\hline ANNAMALAINAGAR & ANN & $11^{\circ} 22^{\prime} \mathrm{N}$ & $79^{\circ} 41^{\prime} \mathrm{E}$ & $1.77 \mathrm{~N}$ & $151^{\circ} .20$ & 3.4 \\
\hline HYDERABAD & HYB & $17^{\circ} 25^{\prime} \mathrm{N}$ & $78^{\circ} 33^{\prime} \mathrm{E}$ & $7.86 \mathrm{~N}$ & $150^{\circ} .69$ & 11.2 \\
\hline SHILLONG & SHL & $25^{\circ} 55^{\prime} \mathrm{N}$ & $91^{\circ} 53^{\prime} \mathrm{E}$ & $14.99 \mathrm{~N}$ & $163^{\circ} .95$ & 20.0 \\
\hline JAIPUR & JAI & $26^{\circ} 55^{\prime} \mathrm{N}$ & $75^{\circ} 48^{\prime} \mathrm{E}$ & $17.30 \mathrm{~N}$ & $147^{\circ} .40$ & 23.0 \\
\hline SABHAWALA & SAB & $30^{\circ} 22^{\prime} \mathrm{N}$ & $77^{\circ} 48^{\prime} \mathrm{E}$ & $20.78 \mathrm{~N}$ & $151^{\circ} .34$ & 27.0 \\
\hline TASHKENT & $\mathrm{TKT}$ & $41^{\circ} 20^{\prime} \mathrm{N}$ & $69^{\circ} 37^{\prime} \mathrm{E}$ & $32.51 \mathrm{~N}$ & $145^{\circ} .52$ & 41.4 \\
\hline
\end{tabular}

sessing the occurrence of focus of the current system on a day-to-day basis during d-months. We attempt to depict the abnormal day-to-day variations in the Declination component at equatorial and low-latitude stations during the dmonths of the low solar activity year 1977 .

\section{Observations and Results}

Careful scrutiny of individual quiet day $(\mathrm{Ap} \leq 7)$ variations of the $H$ and $D$ components during the d-months of the year 1977 revealed a highly variable pattern in the dayto-day variation of the Declination component at the equatorial and low-latitude stations in the Indian longitudinal chain. In an attempt to elucidate this component, we examined certain finer aspects of abnormal day-to-day characteristics of the Sq current system. The database used to depict the day-to-day variability is mainly restricted to the average hourly values of the $H$ and $D$ components for 1977 in terms of the availability of data for individual days spanning from the equator to high-latitude regions; the period of the study falls in the solar minimum, similar to the conditions of 1987. The diurnal changes in $D$ and diurnal changes in the eastward field $(Y)$ showed no appreciable difference at low latitudes. Consequently, the data $\Delta D$ (min.) were converted into $\Delta Y$ (nT) for this analysis. Respective station codes with their geographic and geomagnetic co-ordinates are given in Table 1.

Detailed aspects of anomalous behaviour in the $Y$ component $(Y=H \sin D)$ for individual Quiet days $(\mathrm{Ap} \leq 7)$ are examined by classifying the days of each month of the dmonths (November, December, January, February) of 1977 as normal and abnormal days, respectively. Normal days are designated as the Quiet days of a particular month with a well-defined eastward field (positive $Y$ ) in the morning hours, changing over to westward-directed field during the noon hours at most of the low-latitude locations along the Indian longitude. Abnormal days chosen for the study are days representing a dominant westward-directed field (negative $Y$ ) in the morning hours, when a dominant eastward field is expected. On such abnormal days, the predominance of the westward field has also suppressed the morning peak ( $\sim 0900 \mathrm{~h} \mathrm{LT}$ ), unlike the normal day. The number of days
Table 2.

\begin{tabular}{ccc}
\hline d-months & No. of abnormal days & No. of normal days \\
\hline January & 5 & 4 \\
February & 3 & 2 \\
November & 10 & 6 \\
December & 6 & 8 \\
\hline
\end{tabular}

chosen under abnormal and normal variation conditions, respectively, for each of the d-months is given in Table 2 . The nomenclature represented by $\Delta Y$ and $\Delta H$ defines the departure of the hourly values of $Y$ and $H$ from their respective night base. In order to eliminate the difference of the field between a day's midnight level to the next day's level, we applied a non-cyclic correction (Matsushita and Campbell, 1967) for the data set considered in this investigation.

Average diurnal variation curves of $\Delta Y$ for the selected normal days (as designated) and abnormal days for the four individual months of the d-months of 1977 are shown in Fig. 1. Days chosen for the normal and abnormal category of the respective months are used for the average pattern of variation. In terms of the diurnal pattern for normal days (Fig. 1), the positive variation suggesting a distinctly defined eastward field in the morning hours is obvious at all low latitudes, with a maximum occurring around $0900 \mathrm{~h} \mathrm{LT}$ and a minimum occurring in the afternoon hours. Examination of the $\Delta Y$ variation curves of abnormal days reveals the noticeable absence of the prominent eastward field expected in the morning hours. A dominant westward field (negative) is seen to persist during the prenoon hours from the equator to the latitude of Alibag. However, above this there is only a marginal eastward-directed field, which is quite suppressed up to the latitude of Sabhawala. The average curves of $\Delta Y$ at all of the latitudes shown for the abnormal days of January 1977 show that the eastward field has a distinct magnitude towards the late evenings around $1600 \mathrm{~h} \mathrm{LT}$. Furthermore, a month-to-month scrutiny of the individual Quiet days during the d-months revealed the existence of a certain degree of variability in the day-to-day pattern of the Declination component for individual months. This un- 


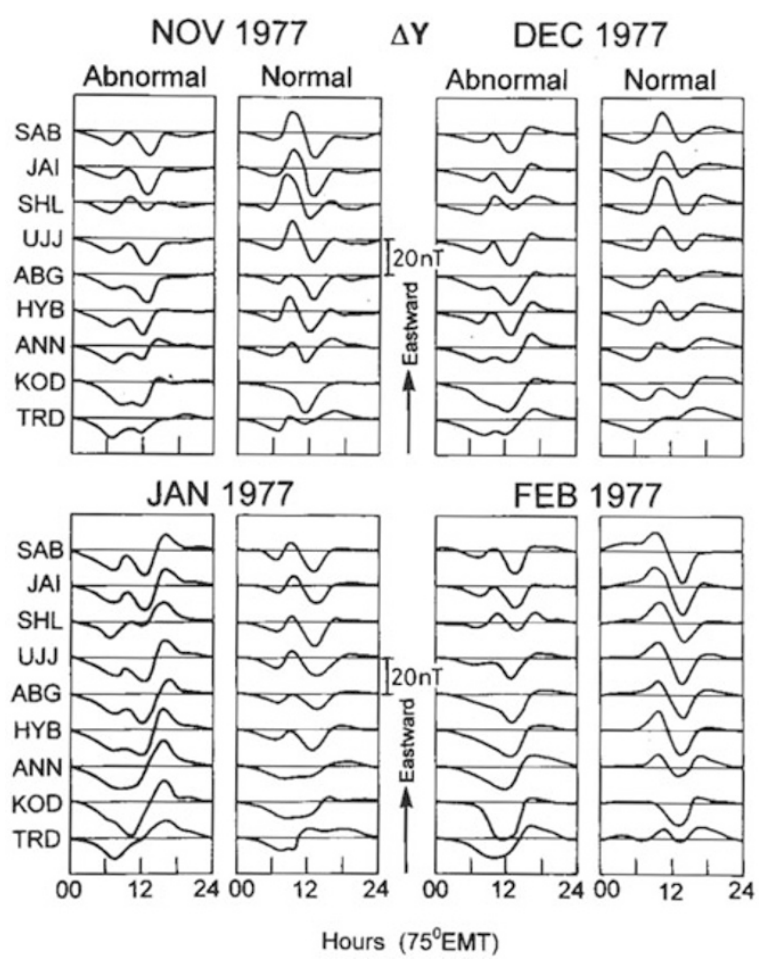

Fig. 1. Average diurnal variation of the east-west $(\Delta Y)$ component for the selected abnormal and normal days of the individual d-months of 1977.

Table 3. The list of selected Quiet days of the d-months.

\begin{tabular}{cccc}
\hline Abnormal days & \multicolumn{2}{c}{ Normal days } \\
\hline Date & Ap & Date & Ap \\
\hline 8 January 1977 & 6 & 2 January 1977 & 5 \\
23 January 1977 & 6 & 26 January 1977 & 4 \\
4 February 1977 & 4 & 16 February 1977 & 6 \\
29 November 1977 & 6 & 3 November 1977 & 4 \\
01 December 1977 & 6 & 19 December 1977 & 3 \\
\hline
\end{tabular}

predictable pattern in the diurnal variation of $Y$ during the d-months of 1977 is illustrated by considering the variations of the $Y$ and $H$ components for selected days with $A p \leq 7$. The list of selected Quiet days of the d-months is given in Table 3.

Latitudinal characteristics of the diurnal variation of $\Delta Y$ and $\Delta H$ for selected pairs of normal and abnormal days of January and February are presented in Figs. 2(a, b) and 3. Referring to Fig. 2(a), 8 January 1977 is seen as an abnormal day, where $\Delta Y$ exhibits a significant negative excursion during the morning hours, with the minimum occurring around 1200 LT, subsequently changing over to a definite positive excursion in the afternoon hours, with a maximum observed around $1500 \mathrm{~h} \mathrm{LT}$ as far up as the Sabhawala latitude; a shift in this maximum is seen at and above Tashkent. Further examination of the $\Delta H$ curves of 8 January 1977 reveals that the persistent northward field at noon is a noticeable feature even up to the latitude of Novosibirsk, suggesting the absence of any trace of the focus position up to this latitude. Referring to the variations on the normal day, 2 January 1977, $\Delta Y$ curves show a marginal increase in the eastward field, with suppressed maxima at $\sim 0900 \mathrm{~h} \mathrm{LT}$ in
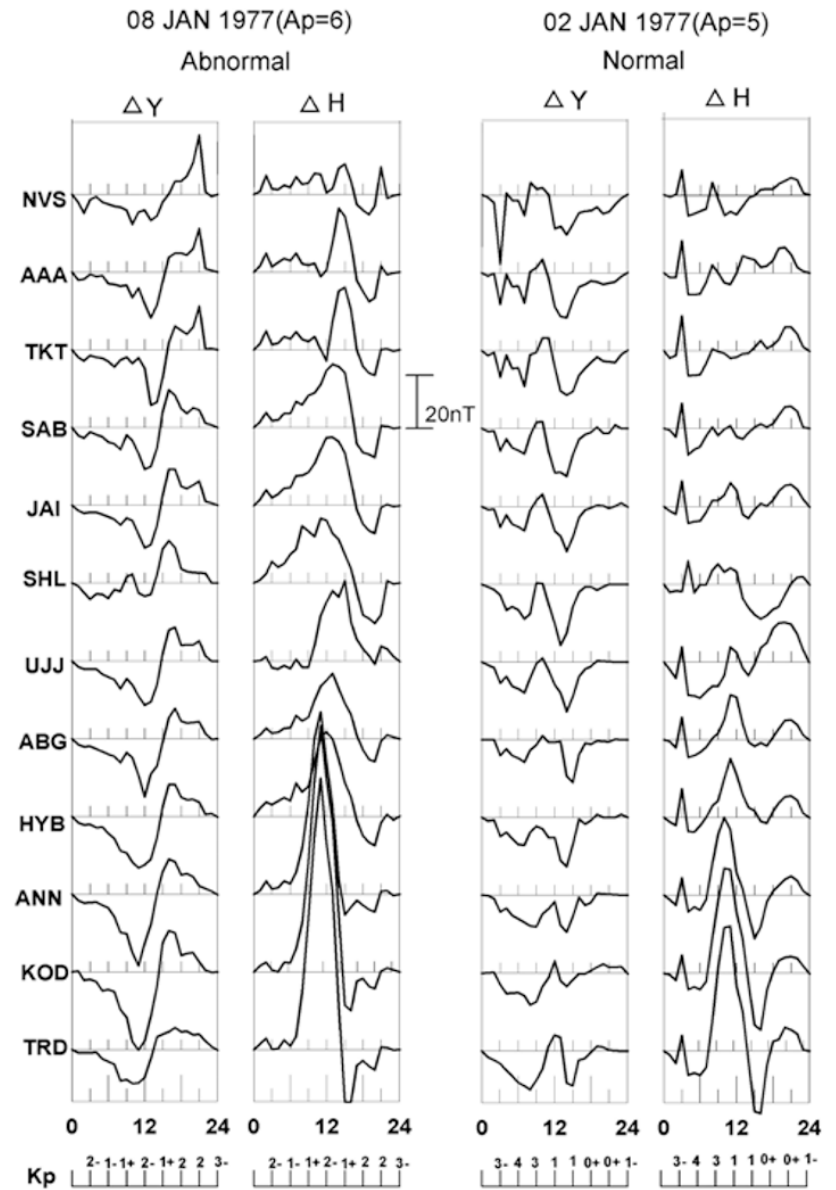

Kp பّำ

$$
\stackrel{\circ}{\text { Hours }(75 \text { EMT) }}
$$

Fig. 2(a). Day-to-day variations of the $\Delta Y$ and $\Delta H$ components for selected abnormal day and normal day pair for January 1977 in the Indian longitudinal sector.

the morning hours and a westward field in the afternoon hours, with the minimum occurring around $1500 \mathrm{~h} \mathrm{LT}$ at all the latitudes. Large prominent fluctuations in the $\Delta H$ and $\Delta Y$ field that can be seen at higher latitudes within the interval $0000 \mathrm{~h}$ LT to $0006 \mathrm{~h} \mathrm{LT}$ are due to the contribution from magnetospheric disturbance, as is evidenced from the three hourly Kp values given below. The influence of the disturbance in the early morning hours could have modified the quiet time current system on this normal day so as to reduce the variation in $H$ towards higher latitude locations. Figure 2(b) shows that 23 January is another abnormal day, representing a similar type of variation in $\Delta Y:$ the presence of a strong westward field suggests the influence of an intense poleward (northward) current during most of the prenoon hours of the day. In terms of the $\Delta H$ and $\Delta Y$ curves for this day, the significant positive amplitude of the northward field and an enhanced eastward-directed field during the afternoon hours are features that persist from equator to the higher latitudes, again revealing the absence of a clear-cut focal position. The $\Delta Y$ variation on 26 January 1977 (normal day), even though there is less influence of the eastward field in the early morning hours, changes the field over to westward at $\sim 1100 \mathrm{~h} \mathrm{LT}$, with a maximum in the after- 


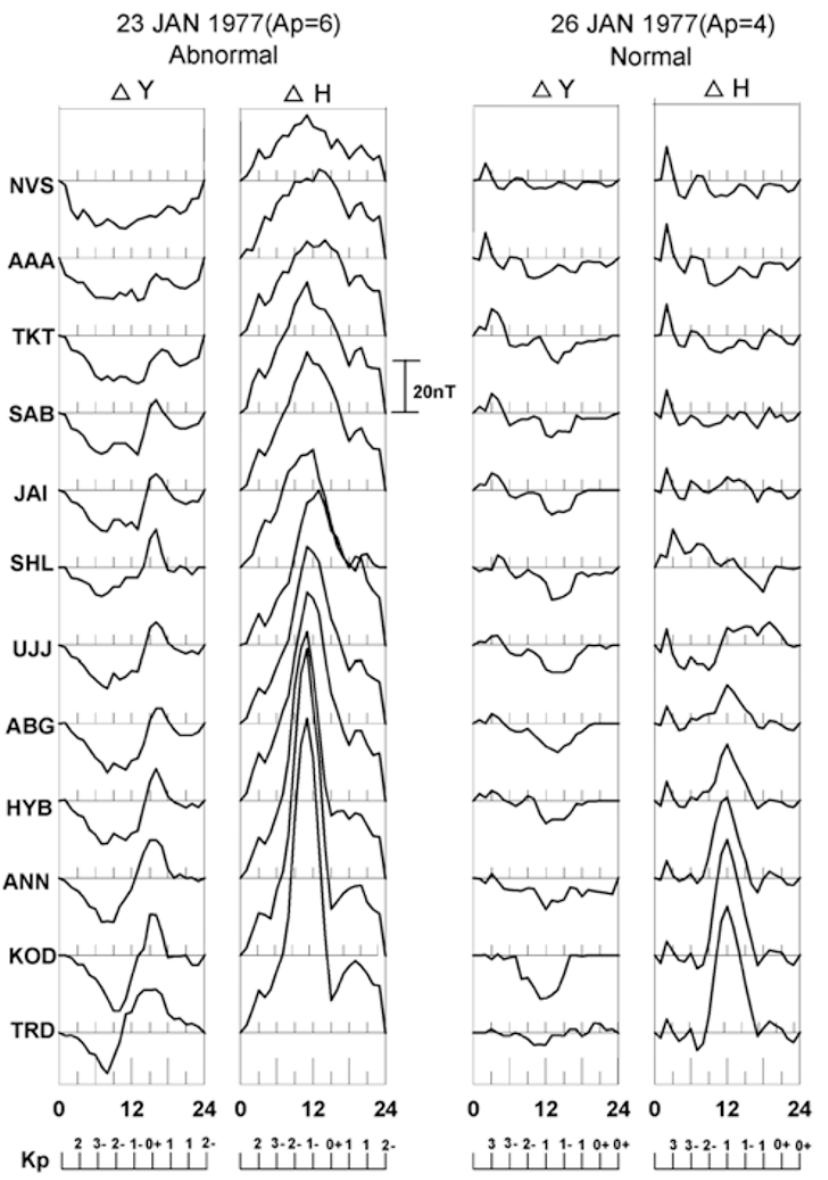

Hours (75 EMT)
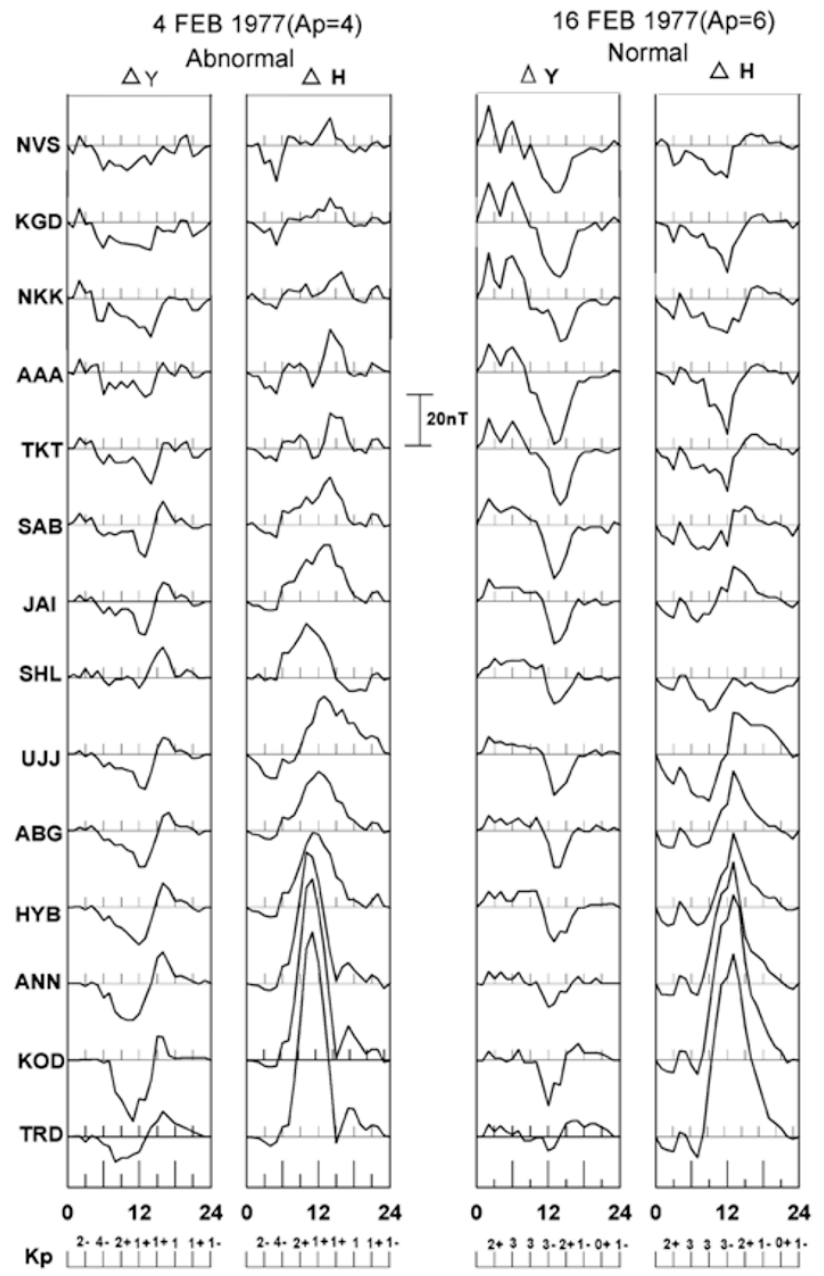

Hours $\left(75^{\circ} \mathrm{EMT}\right)$

Fig. 2(b). Latitudinal variation of $\Delta Y$ and $\Delta H$ on an abnormal day (23 January 1977) and normal day (26 January 1977) for the stations from equatorial (TRD) to high latitudes (NVS) in the Indo-Russian longitude belt.

noon hours. Almost similar to the case of 2 January 1977, the magnitude of diurnal variation with latitude (26 January 1977) on this day is very small compared to that of other normal days. The effect of geomagnetic disturbance during early morning period has had some small influence on the production of very small variations up to the latitude of Novosibirsk, suggesting a change in the current pattern.

Latitudinal variations of the diurnal pattern of $Y$ and $H$ for individual days of February are shown in Fig. 3 by projecting the variations at 14 locations distributed from the equator to high latitudes in the Indo-Russian longitudinal belt. A predominantly negative $\Delta Y$ is noticeable at all the latitudes, from the equator (TRD) to the high-latitude station Novosibirsk, on the abnormal day, 4 February 1977. Defining the $\Delta H$ curves at all latitudes, the absence of focal position is evident; however, a secondary noon minimum in $\Delta H$, deflecting to an evening maximum, is conspicuous at locations from Sabhawala onwards. The $\Delta Y$ variation of the normal day (16 February 1977) exhibits a suppressed level of positive variation that suggests the absence of the expected diurnal maximum in the eastward field during the morning hours, with a subsequent minimum at noontime. The presence of a noticeable magnitude in the $\Delta Y$ maxi- mum is seen during the early morning hours of the day; this magnitude increases perceptibly with latitude. The fluctuating nature of the $H$ and $Y$ fields is seen to persist at all latitudes between $0000 \mathrm{~h}$ and $0009 \mathrm{~h} \mathrm{LT}$, corresponding to the enhanced $\mathrm{Kp}$ values. When the variation of $\Delta H$ is examined, southward turning of the $H$ field during the prenoon hours seems to have started from the latitude of Ujjain, suggesting that the focus must have its location to the south of this latitude. Figure 4 depicts a second pair of days: 29 November 1977 (abnormal day) and 3 November 1977 (normal day). Data were available for individual days of November and December for the chain of stations from the equator to the latitude of Alma-Ata ( $43.4^{\circ} \mathrm{dip}$ ). The diurnal trend in $\Delta Y$ variation on the normal day (3 November 1977) clearly shows the influence of a dominant eastward field, with maximum at $0900 \mathrm{~h} \mathrm{LT}$, changing over to westward-directed field $(\Delta Y=0)$ at $\sim 1000 \mathrm{~h} \mathrm{LT}$ with minima at $1200 \mathrm{~h} \mathrm{LT}$. The $\Delta H$ curves for this day depict an intense magnification of the field at all latitudes up to Sabhawala, suggesting the presence of a strong eastward 


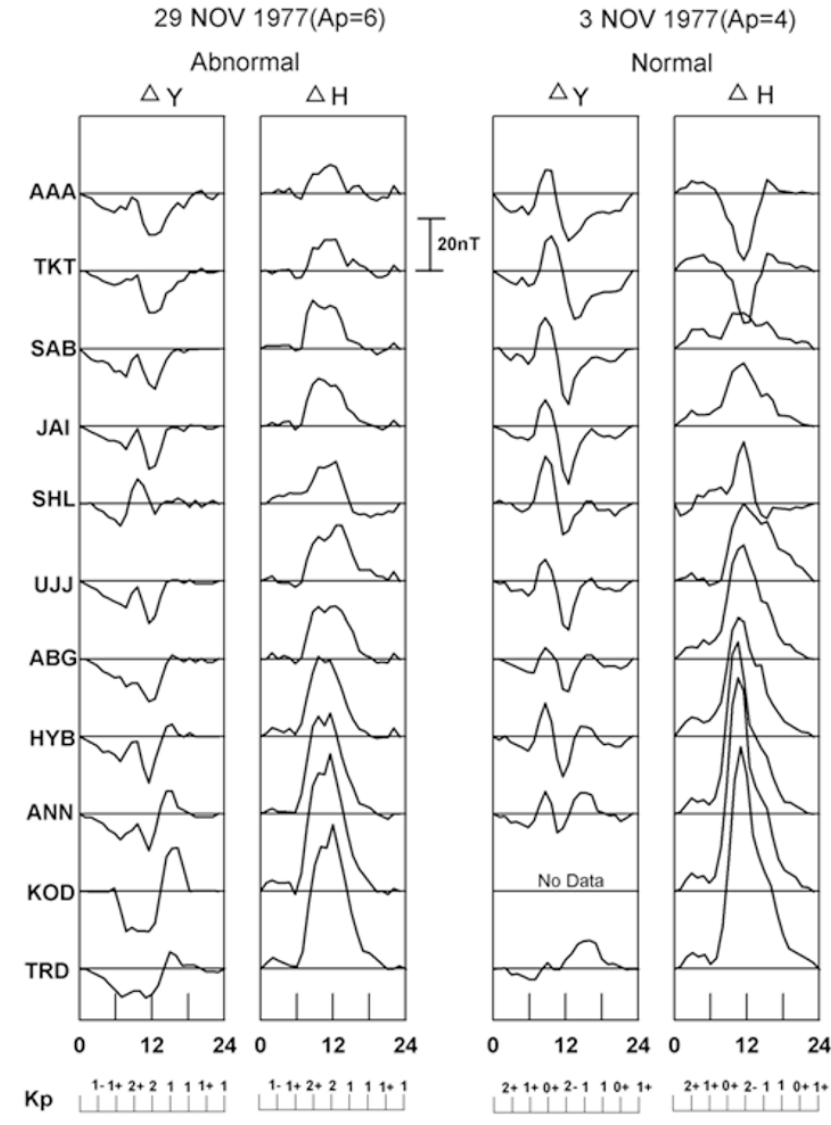

Hours $\left(75^{\circ} \mathrm{EMT}\right)$

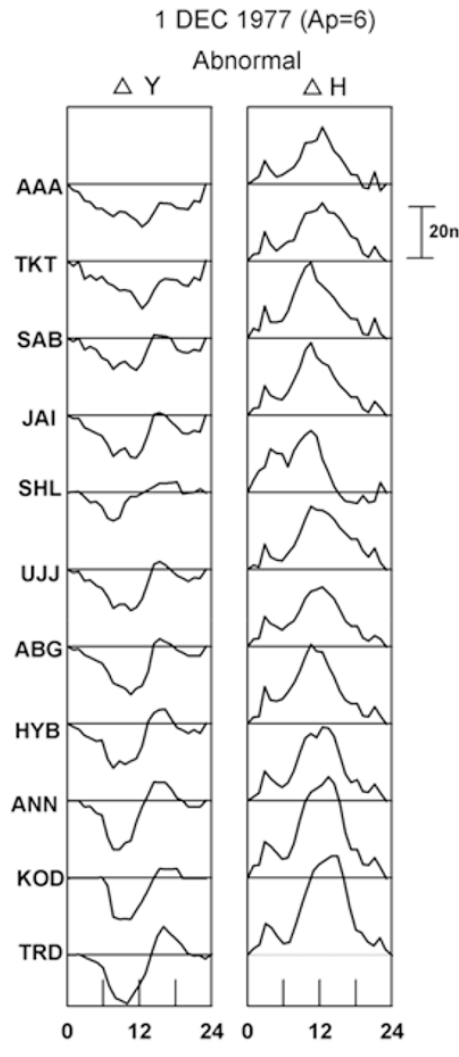

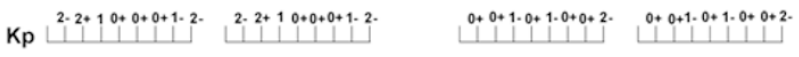

Hours $\left(75^{\circ} \mathrm{EMT}\right)$

Fig. 4. Variation in the $\Delta Y$ and $\Delta H$ components on an abnormal day (29 November 1977) and normal day (3 November 1977) in the dip latitude range of $0^{\circ}$ to $43^{\circ} \mathrm{N}$ from the equatorial (TRD) to midlatitudes (AAA) in the Indo-Russian longitude belt.

Sq current prevalent on this day. The marked reduction in strength with latitude is also evident, and the change-over from a northward to a southward field from Sabhawala to Tashkent suggests that the focal latitude is positioned between these two stations. However, the pattern of $\Delta H$ for the abnormal day (29 November 1977) shows a pronounced noontime peak at all the locations up to Alma-Ata. The $\Delta Y$ for the abnormal day is negative during the prenoon hours at all the locations, suggesting the influence of an intense poleward-directed current prevailing during these hours of day. The cases considered in Fig. 5 also fall in the winter solstice of 1977. Abnormal day, 1 December, shows a dominant westward field at all the latitudes from the equator to Alma-Ata for which the noon maximum in $\Delta H$ variation persisted even up to the latitude of Alma-Ata, suggesting the absence of focus. The pattern of normal day, 19 December 1977 revealed the presence of a northward field up to the focus location (SAB), followed by a southward-directed field type variation at noon at Tashkent and Alma-Ata. $\Delta Y$ variations have followed a well-defined eastward field at all the latitudes during the prenoon hours, followed by the afternoon minimum.

With the aim of delineating the current directions at the low-latitude stations for the abnormal and normal days considered in the present investigation, directional current

Fig. 5. Variation in the $\Delta Y$ and $\Delta H$ components on an abnormal day (1 December 1977) and normal day (19 December 1977) in the dip latitude range of $0^{\circ}$ to $43^{\circ} \mathrm{N}$ from the equatorial (station TRD) to midlatitudes (station AAA) in the Indo-Russian longitude belt. vectors deduced from $\Delta X(X=H \cos D)$ and $\Delta Y(Y=$ $H \sin D$ ) for selected pairs of days are presented in Figs. 6 and 7 and plotted against the local time of the day, and dip latitude. In order to visualize the pattern of the current system at different local times of the day, $\Delta Y$ and $\Delta X$ at different latitudes are utilized by rotating the angular direction by $90^{\circ}$ to indicate the direction and magnitude of the current vector. Increasing magnitude on the $X$-axis is proportional to the magnitude of the eastward-directed current, and increasing angular direction is nearly proportional to the meridional current-directed towards equator. Figure 6(a) and (b) represents the significantly varying current vector pattern for the pair of days of abnormal and normal days for January and February, respectively. For the normal days, 2 January 1977 and 16 February 1977, the vector patterns show a clear indication of the presence of counterclockwise currents, with the active eastward current at the equatorial and low latitudes during the noon hours changing over to westward currents at higher latitudes. The anticipated southward meridional current in the morning hours is not vividly present on all abnormal days, suggesting a distortion in the meridional current system during d-months. A salient feature to discuss regarding the current vectors on the abnormal days, 8 January 1977 and 4 February 1977, is the predominantly eastward-directed (clockwise) vectors 

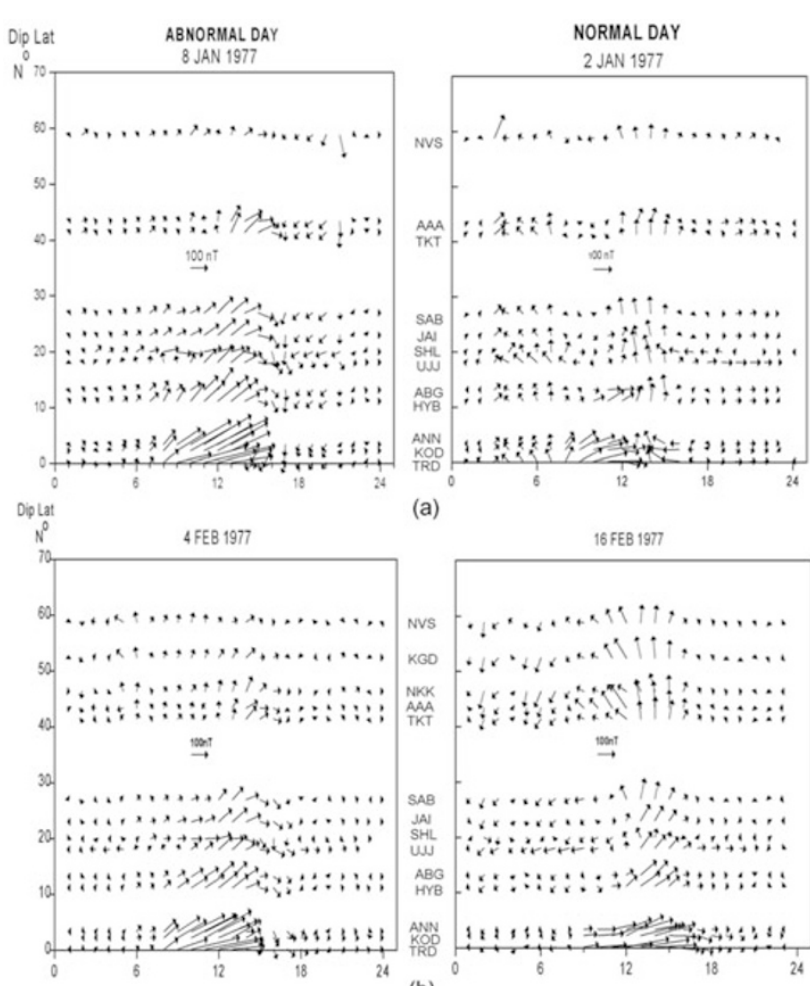

(a)

LOCAL TIME IN HOURS( $\stackrel{\circ}{7}$ EMT)

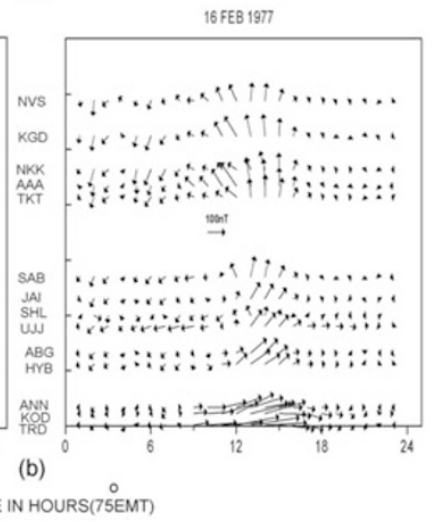

Fig. 6. (a) Local time variations in current vectors on abnormal day, 8 January 1977, and normal day, 2 January 1977. Absence of any trace of the focus formation until the dip latitude of $30^{\circ} \mathrm{N}$ or above is evident for 8 January 1977. Equator-directed vectors are prominent during the evening hours. On 2 January 1977, there was a counter-clockwise variation in the current vectors prevailing towards higher latitudes. (b) 4 February 1977 (abnormal day), persisting eastward-directed vectors at all latitudes during the day hours is prominent. On 16 February 1977 (normal Day), the turning of the eastward-directed vector to a westward-directed one during the noon hours at dip latitudes above $40^{\circ} \mathrm{N}$ explains the pattern of a well-defined focus.

at all latitudes that span the equator to the midlatitude location. The equatorward (southward) meridional current in the evening hours, coupled with the absence of the any trace of focus formation on these abnormal days, is suggestive of the distortion in the Sq current system on a dayto-day basis during the winter months. Similarly, Fig. 7(a) and (b) shows current vectors for pairs of abnormal days (29 November 1977, 1 December 1977) and normal days (3 November 1977, 19 December 1977) in November and December (d-months). The features of the directional current vectors associated with the abnormal days and normal days for these two months also closely resemble the other days shown in Fig. 6.

\section{Discussion and Conclusions}

It is important to take the diurnal pattern of the Sq current system into consideration when assessing the associated contributing parameters, such as local ionospheric conductivity, thermal tidal motions and winds. The association between the highly variable current system and seasonal changes in the semidiurnal wind has been discussed by Stening (1969) and Stening (1989). Based on a simulation study of ionospheric electric field and geomagnetic field

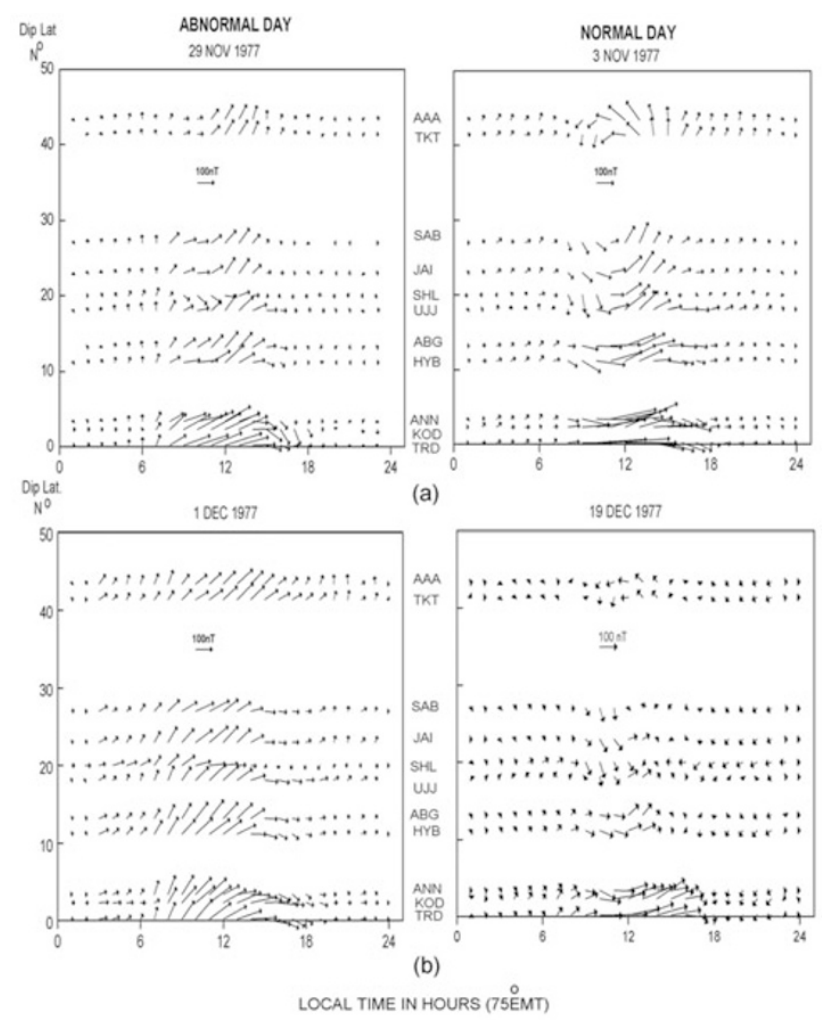

Fig. 7. (a) Abnormal day, 29 November 1977, clearly exhibits the disintegration of the Sq current loop along the Indo-Russian longitude in contrast to the normal day signature of counter-clockwise current vectors, as seen from the current vector directions on 3 November 1977. (b) Abnormal day, 1 December 1977, presents the clockwise pattern of the current direction as opposed to the formation of counter-clockwise current vectors on normal day 19 December 1977.

variation, Takeda et al. (1987) concluded that the semidiurnal tidal winds contribute about one half of the strength of the total Sq current during low solar activity. Their suggestion - that modification of the Sq current generates a clockwise current vortex on the dusk side-seems to be true for the days that we have presented as abnormal days in our study (Figs. 6, 7). Departing from the idea of studying the seasonal or solar cycle characteristics of the current system, we have mainly focused on the day-to-day variability in the $\mathrm{Sq}$ focus as inferred from the variation characteristics of the $\Delta Y$ component at the low-latitude chain of stations in the Indian longitude for the low solar activity year 1977; these are similar to the average features observed for the winter months of 1987 (Alex et al., 1992). Based on their studies of long sequences of Quiet day magnetic field variations, Walker and Kannangara (1982) noted distinct semi-diurnal variations in the $H$ component at midlatitudes $\left(31^{\circ}-43^{\circ} \mathrm{N}\right)$ on certain days of the December solstice.

Different school of thoughts exist with respect to explaining the distortions in the Sq current system and its variability during winter months. Arora et al. (1980) attributed the abnormal current vectors in the d-months in the Indian region to the resultant effect of the action of ionospheric dynamo and the electrostatic fields transmitted from the summer hemisphere to the winter hemisphere. Walker and Kannangara (1982) proposed that the electric field transference between the two hemispheres and the field-aligned currents 
gave rise to the formulation of a current system similar to that seen in the southern hemispheric latitudes. Takeda (1990) compared the estimated equivalent current system with the observed pattern and suggested that the invasion of the current vortex from the summer hemisphere to the winter hemisphere is due to a field-aligned current generated by the asymmetry in the ionospheric dynamo. Day-to-day variability in the regular variation of the magnetic field $S_{\mathrm{R}}$ was illustrated by studying the equivalent electric current system through the analysis of magnetic data and incoherent scatter measurements at Saint-Santin by Mazaudier (1993). She explained the day-to-day variability in the regular external currents as being due to the large-but regular-variability in the tidal winds, which could also be found in the magnetic data. The results presented in this paper suggest differing day-to-day variability in the occurrence characteristics of the Sq current system during d-months. This variability corresponds closely with the findings and proposal of Takeda (1990) and Mazaudier (1993). Our results clearly indicate that the signature of the formation of the northern $\mathrm{Sq}$ focus existed on all of the normal days considered, as is evident from the $H$ variation following the expected diurnal pattern of the $Y$ component. On normal days, 2 January $(A p=5), 26$ January $(A p=4)$ and 16 February $(A p=6)$, the effect of geomagnetic disturbance during the morning period seems to have brought out the changes in the expected current pattern. Butcher (1987) reported that the day-to-day variability in the $\mathrm{Sq}(H)$ amplitudes has an obvious relation to the IMF-dependent complicated current system. On the abnormal days, a strong positive variation in $\Delta H$ persisted up to the latitude location of $\sim 55^{\circ} \mathrm{N}$, suggesting the absence of the focus formation in this hemisphere. However, the abnormal characteristics associated with the dayto-day variability in the Declination pattern suggest that this feature is a peculiarity of individual days and is persistent in the winter months. Takeda (2002) studied the correlation between the $\mathrm{Sq}$ amplitude and the electric conductivity of the ionosphere with sunspot activity. He attributed the low Sq amplitude in winter solstices to the difference in the neutral winds in comparison to the magnetic effect of the field-aligned current transfer between two hemispheres.

One important aspect illustrated by our study is the obvious presence of a dominant clockwise current system active on abnormal days, which is in contrast the anticlockwise current system active on normal days. Anomalous day-today variability in the development pattern of the Sq current is quite evident from the variations in $\Delta Y$ on the abnormal days, especially on the abnormal quiet days of January and February (8 January, 23 January, 4 February). In contrast to the expected pattern of meridional current contribution to maintain the regular Sq current system, these days exhibited a pattern of poleward (northward) current in the morning hours along with a equatorward (southward)-directed current in the evening hours. From the case studies presented, it is obvious that on the abnormal days of January and February there was an effect of significant magnitude, with the equatorward meridional current in the evening hours around $1800 \mathrm{~h}$ LT producing a clockwise current whorl.
Current density variations with local time for various latitudes were projected by Stening (1989) who introduced the $(2,3)$ mode current system in order to produce changes in the $\Delta Y$ variation on some of the abnormal quiet days in the d-months. Secondary peak observed in the $Y$ field around $1600 \mathrm{~h}$ LT on most of the abnormal days were attributed to the contribution from currents of the $(2,3)$ mode resembling the north-south current flow pattern of the "invasion" from the southern hemisphere to the northern one, as discussed by Stening (1989). More detailed aspects of the dayto-day anomaly in the Sq current system with reference to the meridional current pattern have yet to be proved, particularly with respect to its variability with longitude.

\section{References}

Alex, S., L. Jadhav, and D. R. K. Rao, Complexities in the variation of Declination component (D) of the Geomagnetic Field in the Indian region, Geol. Soc. India Mem., 24, 263-274, 1992.

Arora, B. R., D. R. K. Rao, and N. S. Sastri, Latitudinal variations of geomagnetic solar and lunar tides in the Indian region, Proc. Indian Acad. Sci., 89, 333-346, 1980.

Butcher, E. C., Currents associated with abnormal quiet days in $\mathrm{Sq}(\mathrm{H})$, Geophys. J. R. Astr. Soc., 88, 111-123, 1987.

Campbell, W. H. and S. Matsushita, Sq currents: A comparison of quiet and active year behaviour, J. Geophys. Res., 87, 5305-5308, 1982.

Campbell, W. H., E. R. Schiffmacher, and B. R. Arora, Quiet-time ionospheric currents near $75^{\circ} \mathrm{E}$ longitude, (Abstract) EOS, 72, Suppl. 357, 1991.

Campbell, W. H., B. R. Arora, and E. R. Schiffmacher, External Sq currents in the India- Siberia region, J. Geophys. Res., 98, 3741-3752, 1993.

Hasegawa, M., On the position of the focus of the Geomagnetic Sq current system, J. Geophys. Res., 65, 1437-1447, 1960.

Matsushita, S. and W. H. Campbell, Physics of geomagnetic phenomena, vol. 1 ( New York: Academic press), 302, 1967.

Mazaudier, C., On the relationship between ionospheric phenomena and the day-to-day variability of the earth magnetic field regular variation " $S_{\mathrm{R}}$ " Advances made during the two last decades with the incoherent scatter sounder network results, Trends Geophys. Res., 2, 397-421, 1993.

Oyama, K. I., M. A. Abdu, A. Piel, and H. Thiemann, What is happening near Sq Focus?, Adv. Space Res., 26, 1263-1266, 2000.

Patil, A., B. R. Arora, and R. G. Rastogi, Daily variations of the geomagnetic field near the focus of Sq current system in Indian longitude, Proc. Indian Acad. Sci., 92, 239-245, 1983.

Rastogi, R. G., Disintegration of the ionospheric Sq Loop system during winter solstices along $75^{\circ}$ E longitude, Ann. Geophys., 11, 40-46, 1993.

Schlapp, D. M., World-wide morphology of day to day variability of Sq, J. Atmos. Terr. Phys., 30, 1761-1776, 1968.

Stening, R. J., An assessment of the contributions of various tidal winds to the Sq current system, Planet. Space Sci., 17, 889-908, 1969.

Stening, R. J., A calculation of ionospheric currents due to semidiurnal antisymmetric tides, J. Geophys. Res., 94, No. A2, 1525-1531, 1989.

Takeda, M., Geomagnetic field variation and the equivalent current system generated by an ionospheric dynamo at the solstice, J. Atmos. Terr. Phys., 52, 59-67, 1990.

Takeda, M., The correlation between the variation in ionospheric conductivity and that of the geomagnetic Sq field, J. Atmos. Solar-Terr. Phys., 64, 1617-1621, 2002.

Takeda, M. and Y. Yamada, Simulation of ionospheric electric fields and geomagnetic field variation by the ionospheric dynamo for different solar activity, Ann. Geophys., 5A, 429-434, 1987.

Tarpley, J. D., Seasonal movement of the Sq current foci and related effects in the equatorial electrojet, J. Atmos. Solar-Terr. Phys., 35, 1063-1071, 1973.

Walker, G. O. and S. I. Kannangara, A study of quiet day magnetic field variations in East Asia at sunspot minimum, Ann. Geophys., 38(3), 271282,1982 .

S. Alex (e-mail: salex@iigs.iigm.res.in) and M. M. Jadhav 